\title{
Diagnóstico del cáncer de próstata mediante biopsia ampliada de 24 cilindros
}

\author{
A. Rodríguez Alonso, A. González Blanco, S. Pita Fernández**, G. Suárez Pascual, \\ C. Bonelli Martín, J. Lorenzo Franco, J.C. Álvarez Fernández*, M.A. Cuerpo Pérez \\ Servicios de Urología y *Anatomía Patológica. Hospital Arquitecto Marcide, Ferrol, La Coruña. \\ **Unidad de Epidemiología Clínica y Bioestadistica. Hospital Juan Canalejo, La Coruña.
}

Actas Urol Esp 2005; 29 (10): 934-942

\section{RESUMEN}

DIAGNÓSTICO DEL CÁNCER DE PRÓSTATA MEDIANTE BIOPSIA AMPLIADA DE 24 CILINDROS

Objetivo: Determinar el rendimiento diagnóstico de la biopsia prostática (BP) ampliada en el cáncer de próstata $(\mathrm{CP})$ y las variables que influyen en su positividad.

Material y métodos: Pacientes (n=147) sometidos a BP de 24 cilindros, en el Hospital Arquitecto Marcide, Ferrol, La Coruña, entre diciembre 2002-septiembre 2004. Los criterios de inclusión fueron: pacientes $\leq 70$ años con una o más BP negativas o $\leq 75$ años con dos o más BP negativas. Se realizó análisis univariado mediante test $\mathrm{X}^{2}$ en variables cualitativas y test t-Student y U de Mann-Whitney, en variables cuantitativas y análisis de regresión logística para determinar variables relacionadas con la positividad de BP ampliada.

Resultados: 60 pacientes (40,82\%) presentaron CP. Entre los pacientes con BP negativa y positiva, se observaron diferencias significativas en volumen prostático, relación PSA libre/total en la primera BP, relación PSA libre/total en la BP ampliada, PSA-densidad en la BP ampliada y existencia de prostatitis crónica en BP previas. En el análisis multivariado se comprobó que la densidad de PSA y la existencia de prostatitis crónica en BP previas, predecían de forma independiente la positividad de la BP ampliada.

Conclusiones: La BP ampliada permite detectar CP en el 40,82\% de los pacientes con BP previamente negativas. El incremento de la densidad de PSA se asocia una mayor probabilidad de CP, mientras que la existencia de prostatitis crónica en las BP previas reduce significativamente la probabilidad de $\mathrm{CP}$, en la BP ampliada.

Palabras clave: Próstata. Biopsia. Neoplasia de próstata. Adenocarcinoma. Diagnóstico.

\section{ABSTRACT \\ PROSTATE CANCER DIAGNOSIS USING 24 CORES EXTENDED BIOPSY}

Objective: To determine the diagnostic performance of extended prostatic biopsy (PB) in prostate cancer (PC) and variables affecting positivity.

Materials and Methods: Patients $(n=147)$ underwent 24 cylinder PB at the Arquitecto Marcide Hospital, Ferrol, La Coruña, between December 2002-September 2004. Inclusion criteria were the following: patients aged $\leq 70$ with one or more negative PB or aged $\leq 75$ with two or more negative PB. An univariate analysis was carried out using the $\mathrm{X}^{2}$ test for the qualitative variables and the t-Student and $\mathrm{U}$ Mann-Whitney tests in the case of the quantitative variables, plus a logistical regression analysis in order to identify those variables related to the extended PB positivity.

Results: 60 patients (40.82\%) were identified as having PC. Significant differences were observed in prostatic volume, free/total PSA ratio in the initial PB, free/total PSA ratio in the extended PB, PSA-density in the extended PB as well as the existence of chronic prostatitis in previous PB. During the multivariate analysis it was found that the PSA-density and the presence of chronic prostatitis in previous PB independently predicted the positivity of the extended PB.

Conclusions: Extended PB allows for the detection of PC in $40.82 \%$ of patients with previous negative PB. The increase in PSA density is associated with a greater probability of PC, whilst the existence of chronic prostatitis in prior PB significantly reduces the probability of PC in the extended PB.

Keywords: Prostate. Biopsy. Prostatic neoplasms. Adenocarcinoma. Diagnosis. 
$\mathrm{L}$ a biopsia prostática (BP) sextante descrita por Hodge et al $^{1}$ es la técnica habitualmente utilizada para el diagnóstico histológico del cáncer de próstata (CP), sin embargo esta técnica se asocia a un porcentaje importante de falsos negativos ${ }^{2}$.

Esto supone que en muchas ocasiones surja el dilema de qué hacer con pacientes que han sido sometidos a varias sesiones de BP sextante, con resultado negativo y en los que persiste una sospecha clínica y/o bioquímica de CP.

Para intentar incrementar la sensibilidad de la BP sextante, se han desarrollado esquemas de biopsia incluyendo un mayor número de cilindros, combinando habitualmente la BP sextante con el muestreo de la zona posterolateral, medial y transicional. Estos esquemas de BP se han utilizado en pacientes con una o más BP previas negativas, obteniendo una tasa de detección de $\mathrm{CP}$ superior a la de las BP sextantes de repetición ${ }^{3,4}$.

El propósito de este estudio es determinar la utilidad de nuestro esquema de BP ampliada en la detección del $\mathrm{CP}$, en pacientes previamente biopsiados y los factores relacionados con la positividad de la biopsia.

\section{MATERIAL Y MÉTODOS}

Se realizó un estudio observacional de prevalencia incluyendo a 147 pacientes a los que se le practicó biopsia prostática ampliada, de 24 cilindros, en el período comprendido entre diciembre de 2002 y septiembre de 2004, en el Servicio de Urología del Hospital Arquitecto Marcide, Ferrol, La Coruña.

Los criterios de inclusión en el estudio fueron los siguientes: pacientes de edad igual o inferior a 70 años con una o más BP previas negativas y pacientes de edad igual o inferior a 75 años con 2 o más BP previas negativas, en los que persiste la sospecha clínica o bioquímica de cáncer de próstata (tacto rectal sospechoso, PSA $>10 \mathrm{ng} / \mathrm{ml}$, $\mathrm{PSA}<10 \mathrm{ng} / \mathrm{ml}$ con cociente PSA $1 / \mathrm{t} \leq 20 \%$ y/o PSA densidad $\geq 0,15$ ).

Las biopsias previas fueron de 6 cilindros hasta diciembre de 2001 y de 10 cilindros a partir de enero de 2002.

Técnica de biopsia prostática: Todos los pacientes recibieron profilaxis antibiótica con cipro- floxacino $500 \mathrm{mg} / \mathrm{vo} / 12 \mathrm{~h}$, comenzando la noche anterior a la BP y finalizando 2 días después de la realización de la misma. Se administró un enema de limpieza la mañana de la BP, siendo ésta realizada en todos los pacientes bajo sedación intravenosa, utilizando propofol y remifentanilo.

La toma de muestras fue realizada con el paciente en posición de litotomía dorsal, utilizando un ecógrafo BK Leopard 2001 con un transductor transrectal biplanar de 7,5 MHz. Se efectuó inicialmente un examen ecográfico y una medición del volumen de la glándula prostática. A continuación se realizó la BP con una aguja de 18 gauge, recogiendo 20 cilindros de la zona periférica y 4 de la zona transicional. Los cilindros fueron recogidos de forma sistemática, no realizándose en ningún caso biopsias de áreas ecográficamente sospechosas, ya que diversos estudios han demostrado su escasa utilidad para el diagnóstico de CP órganoconfinado ${ }^{5,6}$. Para realizar la toma de muestras se dividió la zona periférica de cada lóbulo prostático en 3 áreas longitudinales: paramedial, intermedia y posterolateral. La biopsia se inició en el lóbulo derecho y la toma de muestras se efectuó desde la base hasta el ápex en cada una de las áreas descritas. Se tomaron 4 muestras de la zona paramedial, 3 de la zona intermedia, que se corresponderían con las muestras de biopsia sextante descrita por Hodge et $\mathrm{al}^{1}$, y 3 de la zona pósterolateral. La zona de transición fue selectivamente biopsiada, en la proximidad de la línea media, avanzando la aguja de punción hasta el límite de la cápsula quirúrgica prostática, biopsiando de este modo la porción más anterior de la glándula. Los cilindros fueron recogidos siempre en el mismo orden, siguiendo un esquema de numeración preestablecido.

Tras la realización de la BP, los pacientes fueron trasladados al área de reanimación, siendo dados de alta al cabo de varias horas, según la evolución, tras comprobar la micción espontánea y una orina aceptablemente clara. No se colocó sonda vesical de forma sistemática a los pacientes; sólo en caso de no presentar micción espontánea.

Los 24 cilindros obtenidos de cada paciente fueron enviados en recipientes independientes para su estudio anatomopatológico. 
Variables del estudio: De cada paciente se recogieron entre 42 y 57 variables, en función del número de BP previas, siendo las más destacadas: edad, número de $\mathrm{BP}$ previas, tiempo transcurrido entre la primera BP y la BP ampliada, PSA total, PSA libre, PSA libre/total, previos a cada BP, velocidad de PSA, densidad de PSA, datos del tacto rectal (tamaño prostático, sospecha de $\mathrm{CP}$ ), volumen prostático determinado por ecografía transrectal (TRUS), anatomía patológica de cada BP, existencia de neoplasia intraepitelial (PIN) de alto grado en BP previas, Gleason combinado, Gleason primario, número de cilindros positivos, localización topográfica de los cilindros positivos, invasión perineural, invasión vascular, invasión linfática, complicaciones postbiópsicas.

Análisis estadístico: Se realizó un análisis descriptivo de todas las variables incluidas en el estudio. Las variables cuantitativas se expresan como media \pm desviación típica y las variables cualitativas como valor absoluto y porcentaje, con su 95\% IC. Se efectuó un análisis univariado, utilizando el test chi-cuadrado para las variables cualitativas. Se empleó el test de Kolgomorov-Smirnov para determinar la distribución normal de las variables cuantitativas y posteriormente estas fueron analizadas mediante el test $\mathrm{T}$ de Student, en caso de distribución normal y la U de Mann-Whitney, para variables con distribución no normal. Se efectuó asimismo un análisis de regresión logística para determinar qué variables permitían predecir la positividad de la $\mathrm{BP}$ ampliada de forma independiente. El análisis estadístico fue realizado mediante software comercialmente disponible.

\section{RESULTADOS}

La media de edad de los pacientes fue $67,63 \pm 6,06$ años. El tacto rectal era sospechoso de CP en 29 pacientes (19,73\%). Se produjeron complicaciones post-biópsicas en 9 pacientes
$(6,12 \%)$, tratándose en 2 casos $(1,36 \%)$ de complicaciones graves: fiebre y bacteriemia por Escherichia coli, que remitió satisfactoriamente con antibioterapia. En los 7 pacientes restantes $(4,76 \%)$ se produjeron complicaciones leves, tratándose en todos los casos de retención aguda de orina. Se observó hematuria leve en 12 pacientes $(8,16 \%)$, no requiriendo hemotransfusión, ni retraso en el alta, ninguno de ellos.

Otras características clínicas y bioquímicas de los pacientes del estudio se pueden observar en la Tabla 1.

De los pacientes estudiados, 60 presentaron CP $(40,82 \%)$. La mediana de Gleason combinado fue 6 (4-9). La distribución por grupos de diferenciación histológica fue la siguiente: $\mathrm{CP}$ bien diferenciado (Gleason 2-4): 11 pacientes (18,33\%); CP moderadamente diferenciado (Gleason 5-7): 36 pacientes (60\%) y CP mal diferenciado (Gleason 8-10): 13 pacientes $(21,67 \%)$.

El número medio de cilindros afectados por $\mathrm{CP}$ fue $5,15 \pm 4,03$ (1-13). En el $70 \%$ de los casos de CP (42/60), los cilindros positivos correspondian exclusivamente a áreas prostáticas no biopsiadas previamente. Asimismo, las biopsias de la zona pósterolateral y/o la zona transicional fueron imprescindibles para el diagnóstico de CP en 29 casos (48,33\%). El resto de las características patológicas se encuentran recogidas en la Tabla 2 .
Tabla 1

Características clínicas de los pacientes del estudio.

\begin{tabular}{|c|c|c|c|c|}
\hline Variable & Media & $\begin{array}{c}\text { Desviación } \\
\text { típica }\end{array}$ & Mediana & $\begin{array}{l}\text { Mínimo- } \\
\text { máximo }\end{array}$ \\
\hline Edad $^{1}$ & 67,63 & 6,06 & 69 & $53-75$ \\
\hline № Biopsias previas & 1,47 & 0,73 & 2 & $1-3$ \\
\hline Volumen prostático por TRUS ${ }^{2}$ & 63,36 & 26,05 & 59 & $24,40-128$ \\
\hline $\mathrm{PSA} 1^{\mathrm{a}} \mathrm{BP}^{3}$ & 13,98 & 9,76 & 11,20 & $4,60-46,30$ \\
\hline $\mathrm{PSA} \mathrm{L} / \mathrm{T} 1^{\mathrm{a}} \mathrm{BP}^{4}$ & 15,78 & 6,87 & 14,30 & $6,00-37,70$ \\
\hline PSA BP ampliada ${ }^{3}$ & 15,19 & 12,74 & 11,10 & $5,10-83,80$ \\
\hline PSA L/T BP ampliada ${ }^{4}$ & 13,22 & 7,61 & 10,95 & $1,40-31,90$ \\
\hline Densidad de PSA en BP ampliada & 0,27 & 0,26 & 0,22 & $0,06-1,65$ \\
\hline Velocidad de $\mathrm{PSA}^{5}$ & 1,22 & 2,34 & 0,56 & $0,00-15,29$ \\
\hline $\begin{array}{l}{ }^{1} \text { años } \\
{ }^{2} \text { centímetros cúbicos } \\
{ }^{3} \mathrm{ng} / \mathrm{ml} \\
{ }^{4} \text { porcentaje }(\%) \\
{ }^{5} \mathrm{ng} / \mathrm{ml} / \text { año }\end{array}$ & & & & \\
\hline
\end{tabular}


Tabla 2

Características patológicas de los pacientes del estudio

\begin{tabular}{|c|c|c|c|}
\hline Variable & $\mathbf{N}$ & $\%$ & $95 \%$ IC \\
\hline \multicolumn{4}{|l|}{ Anatomía patológica } \\
\hline - No cáncer de próstata & $87 / 147$ & $59,18 \%$ & $51,09-66,91$ \\
\hline$-\mathrm{HBP}$ & $21 / 147$ & $14,28 \%$ & $9,31-20,65$ \\
\hline$-\mathrm{HBP}+$ prostatitis crónica & $66 / 147$ & $40,90 \%$ & $36,99-52,99$ \\
\hline - Cáncer de próstata & $60 / 147$ & $40,82 \%$ & $33,08-48,90$ \\
\hline \multicolumn{4}{|l|}{ Distribución por estadio clínico } \\
\hline$\bullet \mathrm{Tlc}$ & $47 / 60$ & $78,33 \%$ & $66,60-87,38$ \\
\hline - $\mathrm{T} 2 \mathrm{a}$ & $9 / 60$ & $15,00 \%$ & $7,57-25,74$ \\
\hline - $\mathrm{T} 2 \mathrm{~b}$ & $4 / 60$ & $6,67 \%$ & $2,15-15,30$ \\
\hline \multicolumn{4}{|l|}{ Lóbulo afectado por el cáncer de próstata } \\
\hline - Derecho & $6 / 60$ & $10,00 \%$ & $4,15-19,63$ \\
\hline - Izquierdo & $18 / 60$ & $30,00 \%$ & $19,44-42,44$ \\
\hline - Ambos & $36 / 60$ & $60,00 \%$ & $47-26-71,78$ \\
\hline Invasión perineural & $7 / 60$ & $11,67 \%$ & $5,24-21,71$ \\
\hline Invasión vascular & $4 / 60$ & $6,67 \%$ & $2,15-15,30$ \\
\hline Invasión perilinfática & $3 / 60$ & $5,00 \%$ & $1,28-13,00$ \\
\hline \multicolumn{4}{|l|}{ Cáncer de próstata en función del $\mathrm{n}^{\circ}$ de $\mathrm{BP}$ previas } \\
\hline$\bullet 1 \mathrm{BP}$ & $32 / 89$ & $35,95 \%$ & $26,50-46,30$ \\
\hline - $2 \mathrm{BP}$ & $25 / 39$ & $64,10 \%$ & $48,27-77,90$ \\
\hline - $3 \mathrm{BP}$ & $3 / 19$ & $15,79 \%$ & $4,17-37,21$ \\
\hline \multicolumn{4}{|l|}{$\begin{array}{l}\text { Cáncer de próstata en función del } n^{\circ} \text { de cilindros } \\
\text { de la última BP }\end{array}$} \\
\hline - 6 cilindros & $28 / 78$ & $35,89 \%$ & $25-85-46,97$ \\
\hline - 10 cilindros & $32 / 69$ & $46,37 \%$ & $34,87-58,17$ \\
\hline \multicolumn{4}{|l|}{$\begin{array}{l}\text { Localización de los cilindros con respecto a la BP } \\
\text { sextante }\end{array}$} \\
\hline $\begin{array}{l}\text { - Los cilindros positivos estarian excluidos de } \\
\text { una BP sextante }\end{array}$ & $35 / 60$ & $58 / 33 \%$ & $45,60-70,27$ \\
\hline $\begin{array}{l}\text { - Los cilindros positivos estarían incluidos } \\
\text { en una BP sextante }\end{array}$ & $16 / 60$ & $26,67 \%$ & $16,65-38,88$ \\
\hline - Localización imprecisa & $9 / 60$ & 15,00 & $7,57-25,74$ \\
\hline \multicolumn{4}{|l|}{$\begin{array}{l}\text { Localización de los cilindros en áreas no biopsiadas } \\
\text { previamente }\end{array}$} \\
\hline - Zona pósterolateral y/o transicional & $44 / 60$ & $73,33 \%$ & $61,11-83,34$ \\
\hline - Exclusivamente en zona posterolateral & $17 / 60$ & $28,33 \%$ & $18,04-40,67$ \\
\hline - Exclusivamente en zona transicional & $2 / 60$ & $3,33 \%$ & $0,56-10,57$ \\
\hline $\begin{array}{l}\text { - Simultáneamente zona posterolateral y zona } \\
\text { transicional, sin otras localizaciones } \\
\text { - Zona posterolateral y/o transicional, junto con }\end{array}$ & $10 / 60$ & $16,67 \%$ & $8,78-27,69$ \\
\hline otras áreas no biopsiadas previamente & $13 / 60$ & $21,67 \%$ & $12,61-33,39$ \\
\hline $\begin{array}{l}\text { - Total de cánceres de próstata en áreas no } \\
\text { biopsiadas previamente }\end{array}$ & $42 / 60$ & $70,00 \%$ & $57,55-80,55$ \\
\hline
\end{tabular}

de prostatitis crónica en BP previas, se comprobó que las variables con capacidad para predecir de forma independiente la positividad de la BP ampliada eran la densidad de PSA y la existencia de prostatitis crónica en las BP previas. De esta forma, los pacientes con una mayor densidad de PSA tienen una mayor probabilidad de presentar $\mathrm{CP}$, mientras que la existencia de prostatitis crónica en las BP previas, se asocia a una probabilidad significativamente menor de CP en la BP ampliada (Tabla 4).

La probabilidad de detectar CP se incrementa linealmente con la densidad de PSA, observando asimismo que los pacientes con prostatitis crónica en las BP previas presentan una menor probabilidad de CP (Figura 1).

\section{DISCUSIÓN}

En 1989 Hodge et al. describieron la técnica de biopsia prostática sextante, que posteriormente se popularizó en todo el mundo convirtiéndose en el "gold standard" para el diagnóstico histológico del CP. Dicha técnica fue diseñada para recoger 6 muestras randomizadas de la zona periférica de la próstata, en el plano parasagital $^{1}$.

Limitar a seis el número de muestras recogidas tiene la ventaja de minimizar la morbilidad de la técnica; sin embargo, en la actualidad se estima que la BP sextante efectúa un

En la Tabla 3 se observan las diferencias encontradas en el análisis univariado, entre los pacientes sin y con CP.

Ajustando por edad, PSA en la BP ampliada, velocidad de PSA, densidad de PSA y existencia infra-muestreo de algunas próstatas ${ }^{7,8}$, y es una técnica insuficiente para detectar todos los CP clinicamente significativos. ${ }^{9,10}$

La tasa de falsos negativos de la BP sextante es del 14-35\%, según diversos estudios ${ }^{1,11-14}$. 
Tabla 3

Análisis univariado para detectar diferencias entre los pacientes sin y con cáncer de próstata

\begin{tabular}{|c|c|c|c|}
\hline & & \multicolumn{2}{|c|}{ Media \pm DT } \\
\hline Edad $^{1}$ & & \multicolumn{2}{|c|}{$67,59 \pm 6,28$} \\
\hline TRUS ${ }^{2}$ & & \multicolumn{2}{|c|}{$68,63 \pm 27,33$} \\
\hline PSA- $1^{\mathrm{a}} \mathrm{BP}^{3}$ & & \multicolumn{2}{|c|}{$12,16 \pm 6,50$} \\
\hline $\mathrm{PSA} \mathrm{L} / \mathrm{T}-1^{\mathrm{a}} \mathrm{BP}^{4}$ & & \multicolumn{2}{|c|}{$17,15 \pm 7,57$} \\
\hline PSA - BP ampliada & & \multicolumn{2}{|c|}{$13,89 \pm 7,23$} \\
\hline PSA L/T - BP ampl & & \multicolumn{2}{|c|}{$14,56 \pm 7,27$} \\
\hline PSA densidad & & \multicolumn{2}{|c|}{$0,22 \pm 0,11$} \\
\hline Tiempo transcurric & & \multicolumn{2}{|c|}{$30,61 \pm 21,27$} \\
\hline Diferencia de $\mathrm{PSA}^{6}$ & & \multicolumn{2}{|c|}{$5,02 \pm 4,99$} \\
\hline PSA velocidad ${ }^{7}$ & & \multicolumn{2}{|c|}{$1,03 \pm 1,00$} \\
\hline № BP Previas & & \multicolumn{2}{|c|}{$1,45 \pm 0,81$} \\
\hline \multicolumn{4}{|c|}{$\begin{array}{l}\text { Induración prostática al tacto } \\
\text { Prostatitis crónica }^{8}\end{array}$} \\
\hline \multicolumn{4}{|c|}{$\begin{array}{l}5 \text { tiempo en meses entre la } 1^{\text {a }} \text { BP y la BP ampliada } \\
{ }^{6} \text { diferencia de PSA entre la } 1^{\text {a }} \text { BP y la BP ampliada } \\
{ }^{7} \mathrm{ng} / \mathrm{ml} / \text { año } \\
8 \text { existencia de prostatitis crónica en BP previas }\end{array}$} \\
\hline \multicolumn{4}{|c|}{$\begin{array}{l}\text { Modelo de regresión logística, ajustando por diversas } \\
\text { covariables }\end{array}$} \\
\hline Variable & OR & $95 \% I C$ & $\mathbf{p}$ \\
\hline Edad & 0,98 & $0,92-1,04$ & 0,650 \\
\hline PSA BP ampliada & 0,96 & $0,89-1,04$ & 0,349 \\
\hline PSA densidad & 6,95 & $2,02-239,51$ & 0,019 \\
\hline PSA velocidad & 0,89 & $0,67-1,20$ & 0,475 \\
\hline Prostatitis crónica & 0,44 & $0,20-0,96$ & 0,040 \\
\hline
\end{tabular}

Estos falsos negativos se pueden calcular basándose en los resultados de las BP repetidas o bien mediante modelos informáticos de simulación ${ }^{2}$.

La BP sextante presenta una baja sensibilidad y se estima que la probabilidad de repetir una biopsia prostática en un paciente en el que persiste la sospecha clínica de CP tras una primera BP negativa, es del 13-41\%, alcanzando el 60\% si existe PIN ${ }^{11}$.

La tasa de detección de CP en las BP sextantes de repetición es variable. Así Keetch et al. comunicaron que la detección de $\mathrm{CP}$ en pacientes

con PSA $>10$, en la $1^{\underline{a}}, 2^{-\underline{a}}$ y $3^{\text {a }}$ BP era del $34 \%$, $19 \%$ y $8 \%$, respectivamente ${ }^{8}$. Por su parte, Djavan et al. presentaron una tasa de detección de CP en la $1^{\mathrm{a}}, 2^{\mathrm{a}}, 3^{\mathrm{a}}$ y $4^{\mathrm{a}}$ BP del $22 \%, 10 \%, 5 \%$ y $4 \%$, respectivamente ${ }^{15}$. Roehl et al. detectaron $\mathrm{CP}$ en el $29 \%, 17 \%, 14 \%, 11 \%, 9 \%$ y $7 \%$, en las BP sucesivas ${ }^{16}$.

En un intento de incrementar la sensibilidad de la BP sextante, se han desarrollado esquemas con un mayor número de cilindros, que se emplean en las biopsias de repetición. Algunos autores, considerando las localizaciones más frecuentes de los tumores en la próstata, realizan un muestreo de la zona periférica posterolateral y de la zona transicional anterior ${ }^{4,11-13,17,18}$. Otros esquemas realizan un muestreo volumen-dependiente, adaptando el número de cilindros al volumen prostático $^{2,7,11,13}$. Finalmente, algunos autores efectúan una toma de muestras transuretral, utilizando el asa del resector ${ }^{12}$.

Los resultados de los estudios que realizan BP ampliada a poblaciones de pacientes con una o más $\mathrm{BP}$ sextante negativas, muestran una tasa 


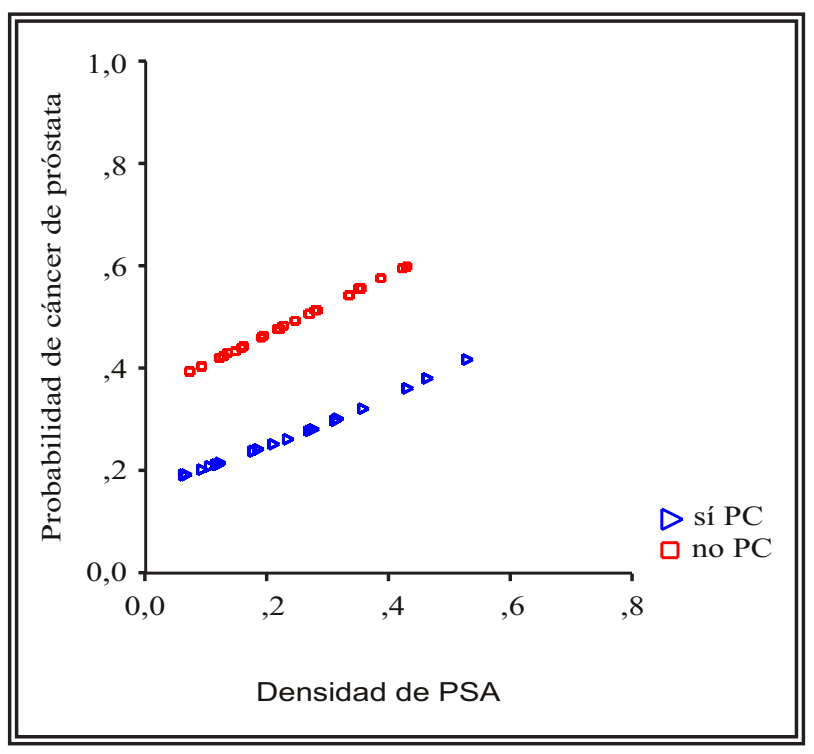

FIGURA 1. Probabilidad de presentar cáncer de próstata en función de la densidad de PSA, ajustando por prostatitis crónica (PC).

de detección de $\mathrm{CP}$ del 13,5\% al 35\%10-13,18. Los esquemas de biopsia y el número de cilindros recogidos es bastante variable, por lo que los resultados son difícilmente comparables entre sí. En cualquier caso, la tasa de detección de $\mathrm{CP}$ en la BP ampliada es generalmente mayor que si se realiza una BP sextante repetida.

En nuestro estudio, la tasa de detección de CP fue del $40,82 \%$. Jones et $\mathrm{al}^{3}$ y Rabets et $\mathrm{al}^{4}$, con un esquema de BP similar al nuestro, obtienen una tasa de CP del 33\% y 29\%, respectivamente.

Nuestra tasa de detección de CP es similar e incluso superior a la observada en algunas series en la primera BP sextante. Este hecho y la evidencia de que la tasa de detección de $\mathrm{CP}$ va decreciendo a medida que se repiten las BP sextantes ${ }^{8,15,16}$, nos hace pensar que las BP sextantes repetidas múltiples siguen presentando un elevado índice de falsos negativos.

En nuestro estudio, el número medio de BP realizadas antes de la $\mathrm{BP}$ ampliada fue 1,52. Si analizamos la tasa de detección de CP en función del número de BP previas, observamos una detección de $\mathrm{CP}$ del 36,67\% en pacientes con una BP previa y del $66,67 \%$ con dos BP previas. Este dato es altamente sugestivo de que el número total de cilindros no es el único factor que influye en el hallazgo de un $\mathrm{CP}$, siendo quizás más importante realizar una adecuada selección de las zonas muestreadas, dirigiendo las biopsias hacia aquellas zonas prostáticas que estadísticamente presentan una mayor probabilidad de $\mathrm{CP}^{11}$.

El CP no presenta una distribución topográfica homogénea, ya que el $80 \%$ se localizan en la zona periférica y el $20 \%$ en la zona de transición ${ }^{7,13}$. Asimismo, en cada una de estas dos zonas, existen ciertas áreas en las que los tumores se asientan con mayor frecuencia, según demuestran diversos estudios. Estas áreas son la posterolateral de la zona periférica y la anterior parasagital de la zona transicional ${ }^{7,19}$.

El área más posterolateral contiene exclusivamente tejido de zona periférica y según diversos estudios, las biopsias dirigidas hacia esta zona son las que aportan un mayor rendimiento diagnóstico en pacientes con BP previas negati$\operatorname{vas}^{2,7,13}$. Algunos autores, como Stamey et al., considerando estos hallazgos, sugieren incluso lateralizar las biopsias del esquema sextante tradicional ${ }^{20}$.

La práctica de biopsias de la zona transicional no se recomienda de forma rutinaria ${ }^{14}$, aunque algunos autores consideran que pueden incrementar la detección de $\mathrm{CP}$ en pacientes con PSA elevado, tacto rectal normal y BP previas negati$\operatorname{vas}^{2}$.

Comprobamos en nuestro estudio que el mayor rendimiento diagnóstico se obtiene con las biopsias de la zona posterolateral, que fueron imprescindibles para establecer el diagnóstico de $\mathrm{CP}$ en 17 pacientes $(28,33 \%)$. Globalmente, el muestreo de la zona posterolateral y/o la zona transicional permitió el diagnóstico de CP en 29 pacientes $(48,33 \%)$.

Teniendo en cuenta estos hallazgos, creemos que el muestreo de la zona transicional debe incluirse en los esquemas de BP ampliada.

Es difícil determinar qué pacientes se van a beneficiar de una BP ampliada, ya que en los estudios realizados no se observa un factor unánimemente relacionado con su positividad. Para Stewart et al., la edad es la única variable que permite predecir la existencia de $\mathrm{CP}$ en la $\mathrm{BP}$ ampliada $^{11}$. Borboroglu et al. observan que la velocidad de PSA predice la existencia de $\mathrm{CP}$ en la BP ampliada, de forma que estableciendo como punto de corte un incremento de PSA de 0,75 $\mathrm{ng} / \mathrm{ml} / \mathrm{año}$, se habrían evitado un $37 \%$ de las BP, 
perdiendo sólo un 6\% de los $\mathrm{CP}^{13}$. Keetch et al., por el contrario, utilizando el mismo punto de corte, evitarian un $42 \%$ de BP, pero perderían un $40 \%$ de los $\mathrm{CP}^{8}$.

Morote et al. comunicaron que un $68 \%$ de los hombres con PSA elevado y sin cáncer en la BP, presentaban algún grado de prostatitis crónica y el $8,4 \%$ de prostatitis aguda ${ }^{21}$.

En nuestro estudio se observó que la existencia de prostatitis crónica en las BP previas era un factor independiente en la predicción de $\mathrm{CP}$, de forma que aquellos pacientes con prostatitis crónica en las BP previas tenían una probabilidad significativamente menor de presentar $\mathrm{CP}$ en la $\mathrm{BP}$ ampliada. Otro factor predictivo independiente en nuestro estudio fue la densidad de PSA.

De este resultado se puede deducir que una elevación de PSA ocasionada por un proceso inflamatorio prostático puede alcanzar unos niveles que indiquen la realización de una BP. También se puede desprender que resulta difícil diferenciar una elevación de PSA producida por un proceso inflamatorio y un proceso tumoral. En aquellos pacientes en cuya biopsia se observa prostatitis crónica, se debe tener en cuenta, antes de repetir la BP, que la elevación del PSA puede ser debida al proceso inflamatorio prostático. Por ello recomendamos administrar un ciclo prolongado de antibióticos con buena difusión en tejido prostático antes de indicar una nueva biopsia prostática.

La BP ampliada suele realizarse bajo sedación intravenosa ${ }^{11-13,18}$, aunque algunos autores observan una buena tolerancia a la técnica mediante anestesia local periprostática ${ }^{3}$. Desde nuestro punto de vista, para este tipo de procedimientos deben utilizarse sedación intravenosa, ya que aumenta el confort del paciente sin retrasar significativamente el alta hospitalaria.

La morbilidad de la técnica es leve en la mayoría de las ocasiones, siendo la hematuria autolimitada la complicación más habitual ${ }^{11,18}$. Si consideramos como complicaciones aquellas que requieren un tratamiento invasivo o el ingreso del paciente, en nuestra serie sólo observamos un $6,12 \%$, tratándose en 7 casos $(4,76 \%)$ de retención aguda de orina y en 2 casos $(1,36 \%)$ de bacteriemia.

Una de las principales críticas realizadas hacia la BP con elevado número de cilindros es la posibilidad de diagnosticar un CP clínicamente insignificante o inocuo, y de realizar en consecuencia un sobretratamiento a hombres cuya supervivencia no se vería afectada por esta enfermedad $^{4,7,11,13}$.

Un CP es clínicamente insignificante cuando sin ser tratado específicamente, nunca va a causar la muerte del paciente, ni va a producir síntomas relacionados con el cáncer. Es por lo tanto un CP que puede ser manejado de forma expectante ${ }^{22}$.

En lo que respecta a la detección potencial de CP clínicamente insignificante al aumentar el número de cilindros biópsicos, existen dos cuestiones importantes que debemos contestar. En primer lugar si el aumento del número de cilindros supone un incremento en la detección de $\mathrm{CP}$ clínicamente insignificante y en segundo lugar si el CP llamado clínicamente insignificante no afecta realmente a la supervivencia del enfermo.

Dugan et al. han profundizado en el estudio del CP clínicamente insignificante y lo definen como aquel $\mathrm{CP}$ que no crecería más de $20 \mathrm{cc}$ de volumen en el momento en el que se espera la muerte el paciente, según la esperanza de vida de su región o país, y cuyo Gleason combinado es menor que el dígito de las decenas de la edad del paciente $^{23}$. La adopción de 20 cc como punto de corte, se debe a que en la mayoría de las metástasis del CP se producen en la última duplicación tumoral (crecimiento de 10 a $20 \mathrm{cc}{ }^{24}$.

Stamey et al. establecen el límite de la significación clínica del $\mathrm{CP}$ en $0,5 \mathrm{cc}^{25}$. Epstein et al. dividen los CP menores de 0,5 cc en: tumor insignificante $(<0,2 \mathrm{cc})$ y tumor mínimo (entre $0,2 \mathrm{y}$ $0,5 \mathrm{cc}$ ), estableciendo que los criterios patológicos de tumor mínimo son: volumen $<0,5 \mathrm{cc}$, confinado al órgano y Gleason score $<7^{5,26}$. La mayoría de los autores adoptan 0,5 cc como cifra crítica en la significación clínica ${ }^{4,13,27,28}$.

Desde nuestro punto de vista, definir la significación clínica de un $\mathrm{CP}$ adoptando un volumen tumoral fijo, no es adecuado en una enfermedad en la que la edad del paciente es básica para la decisión terapéutica y la supervivencia cáncerrelacionada. Un CP con Gleason combinado $5 \mathrm{y}$ un volumen de $0,5 \mathrm{cc}$ no tiene la misma trascendencia pronóstica en un hombre de 50 años que en uno de 70. Por otra parte, la adopción del 
volumen tumoral como piedra angular de la significación clínica no es útil ya que la determinación de la significación clínica debe ser efectuada preoperatoriamente, para poder tomar decisiones terapéuticas.

En la actualidad no es posible identificar preoperatoriamente, y de forma segura, a los pacientes con CP clínicamente insignificante, a pesar del intento de algunos autores por definir factores predictivos ${ }^{5,28,29}$. Así, Epstein et al. establecen que el mejor modelo para predecir la existencia de un tumor insignificante en estadio T1c es: PSA densidad menor de 0,15 y ningún hallazgo patológico adverso (Gleason score $<7$, menos de 2 cilindros afectados y ningún cilindro con más del $50 \%$ de afectación) $)^{5,26}$

Epstein et al. utilizan posteriormente otra combinación de parámetros con la que obtienen una mayor capacidad predictiva de CP clínicamente insignificante: relación PSA libre/total mayor o igual al 15\%, menos de 3 cilindros afectados, ningún cilindro con afectación superior al $50 \%$ y Gleason score $<7^{29}$.

Ninguno de estos modelos predictivos puede ser utilizado de forma segura en nuestra serie, en primer lugar porque fueron diseñados para BP sextante y en segundo lugar porque en nuestro estudio 29 pacientes $(19,73 \%)$ fueron clasificados como T2a. No obstante, entre los pacientes clasificados como T1c, sólo 8 de ellos presentaban un Gleason $<7$, y de estos, 2 presentaban invasión perineural en la BP y 4 un PSA $>12 \mathrm{ng} / \mathrm{ml}$. Es cierto que existe un alto grado de discordancia entre el Gleason de la BP y el de la pieza quirúrgica, pero hay que tener en cuenta que en la mayoría de los casos la BP efectúa una infragraduación del Gleason score ${ }^{30,31}$.

El incremento del número de cilindros biópsicos no se asocia, según algunos estudios, a un aumento significativo en la detección de tumores clínicamente inocuos ${ }^{4,9,13,18}$. Tampoco se observa una mayor detección de CP clínicamente insignificante en pacientes con PSA $<4^{32,33}$.

Por otra parte, Singh et al. comprueban que la toma de un mayor número de cilindros incrementa la detección tanto de CP clínicamente insignificante como de CP clínicamente significativo ${ }^{10}$.

La probabilidad de observar CP clínicamente insignificante en las piezas de prostatectomía radical oscila entre el $2 \%$ y el $27 \%$, según las series. En este sentido, Chan et al. ${ }^{9}$ y Gardner et al. ${ }^{34}$ observan que la existencia de criterios de cáncer mínimo en la BP no predice la existencia de un CP clínicamente insignificante en la pieza quirúrgica.

Una limitación de nuestro estudio, es que no hemos realizado análisis de las piezas de prostatectomía radical, ya que no era el objetivo del estudio. Por ello no es posible determinar con exactitud el volumen tumoral y por lo tanto la significación clínica del tumor.

Es posible que la BP ampliada dé lugar a un incremento en la detección de $\mathrm{CP}$ clínicamente insignificante, pero también aumenta la tasa de detección de CP clínicamente significativo en estadios más tempranos. Este hecho justifica plenamente, a nuestro entender, la realización de BP ampliada, ya que los tumores clínicamente insignificantes detectados, pueden ser manejados de forma expectante. Otra cuestión discutible es si los tumores considerados como clínicamente insignificantes son realmente inocuos, ya que algunos estudios demuestran que los tumores de pequeño volumen pueden tener una actividad proliferativa celular y unas características citométricas similares a los de gran volumen ${ }^{35}$.

La ventaja de la BP ampliada es que identificaría a un grupo de pacientes con tumores significativos, que serían subsidiarios de tratamiento curativo, que es el mejor modo de lograr una mayor supervivencia ${ }^{9,18}$. Por otro lado, el aumento del número de cilindros permite diagnosticar antes el $\mathrm{CP}$, en aquellos pacientes con una primera BP falsamente negativa, evitando la repetición innecesaria de biopsias de próstata, y ganando tiempo en el tratamiento del cáncer.

\section{REFERENCIAS}

1. Hodge KK, McNeal JE, Terris MK, Stamey TA. Random systematic versus directed ultrasound guided transrectal core biopsies of the prostate. J Urol 1989;142:71-75.

2. Terris MK. Prostate biopsy strategies: past, present, and future. Urol Clin North Am 2002;29(1):205-212.

3. Jones JS, Oder M, Zippe GD. Saturation prostate biopsy with periprostatic block can be performed in the office. $J$ Urol 2002;168:2108-2110.

4. Rabets JC, Jones JS, Patel A, Zippe GD. Prostate cancer detection with office based saturation biopsy in a repeat biopsy population. J Urol 2004;172:94-97.

5. Epstein JI, Walsh PC, Carmichael M, Brendler CB. Pathologic and clinical findings to predict tumor extent of nonpalpable (stage T1c) prostate cancer. JAMA 1994;271(5):368-374. 
6. Ellis WJ, Chetner MP, Preston SD, Brawer MK. Diagnosis of prostatic carcinoma: the yield of serum prostate specific antigen, digital rectal examination and transrectal ultrasonography. J Urol 1994;152:1520-1525.

7. Vashi AR, Wojno KJ, Gillespie B, Oesterling JE. A model for the number of cores per prostate biopsy based on patient age and prostate gland volume. J Urol 1998;159:920-924.

8. Keetch DW, Catalona WJ, Smith DS. Serial prostatic biopsies in men with persistently elevated serum prostate specific antigen values. J Urol 1994;151:1571-1574.

9. Chan TY, Chan DY, Stutzman KL, Epstein JI. Does increased needle biopsy sampling of the prostate detect a higher number of potentially insignificant tumors?. J Urol 2001;166:2181-2184.

10. Singh H, Canto EI, Shariat SF, Kadmon D, Miles BJ, Wheeler TM, et al. Improved detection of clinically significant, curable prostate cancer with systematic 12-cores biopsy. J Urol 2004;171:1089-1092.

11. Stewart CS, Leibovich BC, Weaver AL, Lieber MM. Prostate cancer diagnosis using a saturation needle biopsy technique after previous negative sextant biopsies. J Urol 2001;166:86-92.

12. Fleshner N, Klotz L. Role of "saturation biopsy" in the detection of prostate cancer among difficult diagnostic cases. Urology 2002;60(1):93-97.

13. Borboroglu PG, Comer SW, Riffenburgh RH, Amling CL. Extensive repeat transrectal ultrasound guided prostate biopsy in patients with previous benign sextant biopsies. $\mathrm{J}$ Urol 2000;163:158-162.

14. Gil Martínez P, Allepuz Losa C, Gil Sanz MJ, Oliva Encina J, Andrés Lázaro V, Valdivia Navarro P, et al. Rebiopsia de próstata. Factores pronósticos del resultado anatomopatológico. Actas Urol Esp 2000;24(7):560-567.

15. Djavan B, Remzi M, Marberger M. When to biopsy and when to stop biopsying. Urol Clin North Am 2003;30(2): 253-262.

16. Roehl KA, Antenor JAV, Catalona WJ. Serial biopsy results in prostate cancer screening study. J Urol 2002;167:24352439.

17. Babaian RJ, Toi A, Kamoi K, Troncoso P, Sweet J, Evans $\mathrm{R}$, et al. A comparative analysis of sextant and an extended 11-core multisite directed biopsy strategy. J Urol 2000;163:152-157.

18. Eskew LA, Bare RL, McCullough. Systematic 5 region prostate biopsy is superior to sextant method for diagnosing carcinoma of the prostate. J Urol 1997;157:199-203.

19. Chen ME, Troncoso P, Johnston D, Tang K, Babaian RJ. Optimization of prostate biopsies using computer based analysis. J Urol 1997;158(6):2168-2175.

20. Stamey TA. Making the most out of six systematic biopsies. Urology 1995;45(1):2-12.

21. Morote J, López M, Encabo G, de Torres IM. Effect of inflammation and benign prostatic enlargement on total and percent free serum prostatic specific antigen. Eur Urol 2000;37(5):537-540.

22. Schröder FH. Screening for prostate cancer. Urol Clin North Am 2003;30(2):239-251.

23. Dugan JA, Bostwick DG, Myers RP, Qian J, Bergstralh EJ, Oesterling JE. The definition and preoperative prediction of clinically insignificant prostate cancer. JAMA 1996; 275(4):288-294.
24. Bostwick DG, Graham SD Jr, Napalkov P, Abrahamsson PA, di Sant'agnese PA, Algaza F. Staging of early prostate cancer: a proponed tumor volume-based prognostic index. Urology 1993;41:403-411.

25. Stamey TA, Freiha FS, McNeal JE, Redwine EA, Whittemore AS. Localizad prostate cancer. Cancer 1993; 71(3): 933-938.

26. Allan RW, Sanderson H, Epstein JI. Correlation of minute $(0,5 \mathrm{~mm}$ or less) focus of prostate adenocarcinoma on needle biopsy with radical prostatectomy specimen: role of prostate specific antigen density. J Urol 2003;170:370372 .

27. Levine MA, Ittman M, Melamed J, Lepor H. Two consecutive sets of transrectal ultrasound guided sextant biopsies of the prostate for the detection of prostate cancer. J Urol 1998;159:471-476.

28. Huland H, Graefen M, Haese A, Hammerer PG, Palisaar J, Pichlmeier U, et al. Prediction of tumor heterogeneity in localized prostate cancer. Urol Clin North Am 2002;29(1): 213-222.

29. Epstein JI, Chan DW, Sokoll LJ, Walsh PC, Cox JL, Rittenhouse $\mathrm{H}$, et al. Nonpalpable stage T1C prostate cancer: prediction of insignificant disease using free/total prostate specific antigen levels and needle biopsy findings. J Urol 1998;160:2407-2411.

30. Gregori A, Vieweg J, Dahm P, Paulson DF. Comparison of ultrasound-guided biopsies and prostatectomy specimens: predictive accuracy of Gleason score and tumor site. Urol Int 2001;66(2):66-71.

31. Djavan B, Kadesky K, Klopukh B, Marberger M, Roehrborn CG. Gleason scores from prostate biopsies obtained with 18-gauge biopsy needles poorly predict Gleason scores of radical prostatectomies specimens. Eur Urol 1998;33(3): 261-270.

32. Sokoloff, MH, Yang XJ, Fumo M, Mhoon D, Brendler CB. Characterizing prostatic adenocarcinomas in men with a serum prostate specific antigen level of $<4.0 \mathrm{ng} / \mathrm{ml}$. BJU Int 2004;93:499-502.

33. Luján M, Paez A, Miravalles E, Fernández I, Llanes L, Berenguer A. Prostate cancer detection is also relevant in low prostate specific antigen ranges. Eur Urol 2004;45: 155-159.

34. Gardner TA, Lemer ML, Schlegel PN, Waldbaum RS, Vaughan ED Jr, Steckel J. Microfocal prostate cancer: biopsy cancer volume does not predict actual tumor volume. Br J Urol 1998;81:839-843.

35. Horninger W, Berger AP, Rogatsch H, Gschwendtner A, Steiner H, Niescher M, et al. Characteristics of prostate cancers detected at low PSA levels. Prostate 2004;58:232237.

Dr. A. Rodríguez Alonso

Servicio de Urología

Hospital Arquitecto Marcide

Ctra. San Pedro de Leixa, s/n

15405 Ferrol, La Coruña

e-mail: arodri68@terra.es

(Trabajo recibido el 20 de abril 2005) 